\title{
Location and Identification of Acoustic Signals Recorded in Power Oil Transformers within the Band of $20-180 \mathrm{kHz}$
}

\author{
A. Olszewska And F. Witos* \\ Department of Optoelectronics, Silesian University of Technology \\ B. Krzywoustego 2, 44-100 Gliwice, Poland
}

\begin{abstract}
Emission acoustic signals, recorded in investigated power oil transformers, have been analyzed in the time, frequency and time-frequency domain. Analysis of each signal has been started by filtration within selected frequency band and subsequently the following quantities have been calculated: spectral power density, phase-time characteristic, averaging phase-time characteristic, short-time Fourier transform spectrograms, signal amplitude distributions, descriptors with acronyms ADC and ADP and thereafter maps of descriptors on lateral walls of transformers can be carried out. Frequency bands applied in order to filtration have been chosen in such a way so that signals coming from different sources (among other things from partial discharges, Barkhausen's effect, oil circulation and outer acoustic disturbances) can be differentiated. The sources have been localized using maps of descriptors calculated for selected frequency bands. The fundamental properties of obtained signals have been determined. Such properties describe: partial discharges, Barkhausen's acoustic effect and other acoustic interferences.
\end{abstract}

PACS: 52.80.-s, 77.22.Jp, 84.70.+p, 43.40.Le, 43.60.Lq

\section{Introduction}

Subject-matter of the article concerns application of an author's method to locate and identify acoustic emission (AE) signals in power oil transformers, coming from different acoustic sources. Such a method includes the basic and advanced description of AE signals, including maps of descriptors.

Results presented in the article are obtained on the basis of analysis relative to AE signals recorded in two transformers of identical construction but being in different technical state resulting from their operation. There were oil power transformers with transformer ratio of $120 / 20 / 6 \mathrm{kV}$ and rated power of 25 MVA. The first (1) transformer was is good technical state whereas the second (2) of them was superheated several times and was disconnected by reason of discharges, which indicates its bad repair.

In order to investigate transformers an author's measuring system called AE DEMA-COMP [1-6] was applied; such a system has been equipped with AE sensors D9241A type, produced by Euro Physical Acoustic Corporation. AE signals have been recorded at chosen measuring points, accessible for measurement. These points create a measuring network on lateral wall surfaces of transformer tanks. Signals were recorded several times

\footnotetext{
* corresponding author; e-mail: fwitos@polsl.pl
}

at each measuring point; such a recording lasted by $2 \mathrm{~s}$ (100 periods of the supply voltage).

At first, recorded signals have been put to filtration within whole range of analyzed frequencies $(20-120 \mathrm{kHz})$ and within selected frequency bands $(20-70 \mathrm{kHz}, 60-$ $120 \mathrm{kHz}$ and $110-200 \mathrm{kHz}$ ). Frequency bands used to filtration of signals were chosen in such a way so we can distinguish signals coming from different sources (among other things from partial discharges, Barkhausen's effect, oil circulation and outer acoustic disturbances). In the second step, for each of AE signals the following quantities have been calculated: basic characteristics, amplitude distributions of signals and corresponded with them descriptors with acronyms ADP, ADC and ADNC [1-6]. Basing on kriging method, the maps of descriptors on lateral walls of transformer tanks have been worked and local maxima of descriptors have been found. These maps, worked up for chosen frequency bands, were used for location and identification of different acoustic sources.

\section{Maps of descriptors and location of sources}

Maps of ADC descriptor calculated for chosen analyzed frequency bands are presented in Figs. 1 and 2 according to transformer 1 and 2. The $X Y$ plane on these maps is a developed lateral wall of the transformer tank. Coordinates of the points on this plane are in centimeters and determined by the following way: values of $Y$ coordinates are calculated from the bottom of the tank, $X$ 
value is zero for the center of tap changer, values of $X$ coordinates increase in direction of higher voltage side (high-voltage winding), values of $X$ coordinates decrease in direction of lower voltage side (low-voltage winding), maximum and minimum values of $X$ coordinates describe points opposite to points describing the center of the tap changer.

Maximum values of the descriptor on the map within $110-200 \mathrm{kHz}$ band determine places where AE waves coming from partial discharges are dominant in the signals.

Maximum values of the descriptor on the map within 20-70 kHz band determine places where areas where in signals AE waves coming from inner acoustic interferences are dominant, on condition that corresponding with them descriptor values in the band of $110-200 \mathrm{kHz}$ are small.

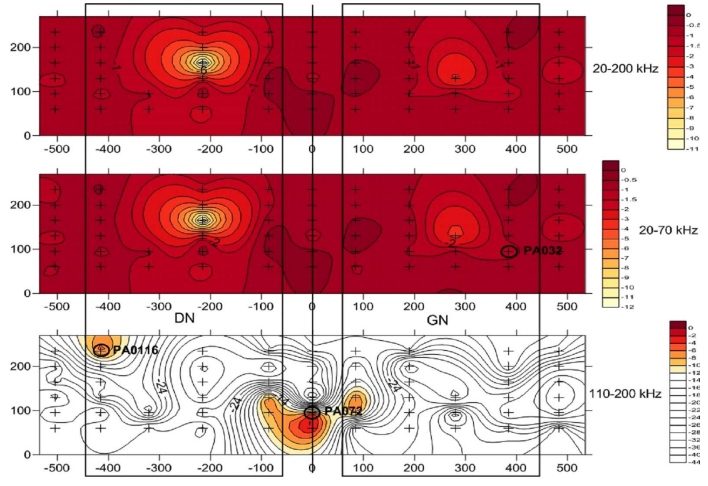

Fig. 1. Maps of ADC descriptor on lateral walls of the tank of transformer 1 within chosen frequency bands. Tank dimensions $(X, Y)$ in centimeters: $X$ - running position along the transformer tank, 0 - the center of the tap changer, positive $X$ values - part of the tank from higher voltage side, negative $X$ values - part of the tank from lower voltage side, +- marked measuring points.

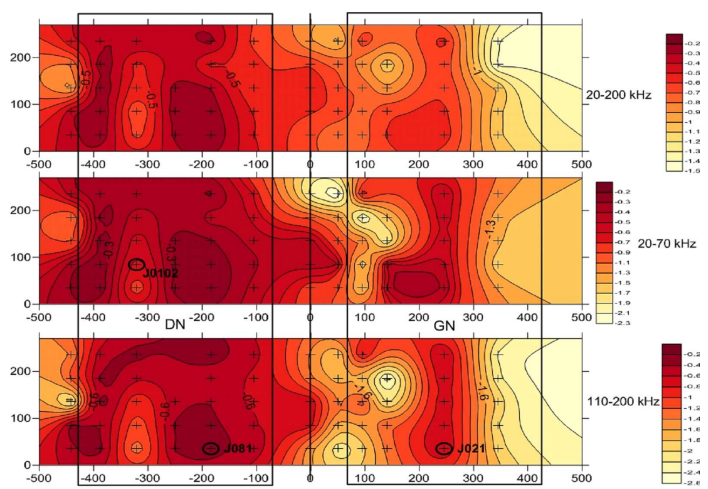

Fig. 2. As in Fig. 1 but for transformer 2 .

Position of sources is located on such distinguished areas by means of modified loudness method [1]. Analysis of maps of ADC descriptor for transformer 1 (Fig. 1) leads to location of partial discharges in the area of the tap changer, and in the area of higher voltage side with coordinates $X=400-420 \mathrm{~cm}, Y=230-250 \mathrm{~cm}$. In the case of transformer 2 (Fig. 2) locations of main partial discharges (PD) sources are as follows:

1. extensive PD sources from higher-voltage side (horizontally $220-270 \mathrm{~cm}$ ) and from lower voltage side (vertically $150-250 \mathrm{~cm}$ ),

2 . the source located from lower voltage side (horizontally $250-350 \mathrm{~cm}$, vertically $230-270 \mathrm{~cm}$ ),

3 . the source located from lower voltage side (horizontally $390-450 \mathrm{~cm}$, vertically $40-90 \mathrm{~cm})$.

\section{Identification of properties of AE signals for different sources}

Analysis of properties of AE signals coming from located areas leaded to distinguish signals describing inner acoustic interferences and partial discharges. Example characteristics for particular types of sources are presented in the next part of the article.

\subsection{Inner acoustic interferences}

Characteristics belonging to basic description of chosen signals recorded in transformers 1 and 2, whose principal source are inner acoustic interferences, are presented in Figs. 3 and 4.

Main frequency band for these signals is $20-40 \mathrm{kHz}$. For signal recorded in transformer 1 (Fig. 3) four main bands appear at the averaging phase characteristic (Fig. 3b). These bands correspond with four fragments situated at the hysteresis loop where effects connected with over-magnetization and principally with Barkhausen's acoustic effect are dominant.

Partial discharges in transformer 2 appear simultaneously with phenomena discussed in Fig. 3, so at the averaging phase characteristic (Fig. 4b) four bands observed previously (Fig. 3b) are connected and created two bands.

\subsection{Partial discharges}

Characteristics belonging to the basic description of signals coming from $\mathrm{PD}$, recorded within transformers 1 and 2, are presented in Figs. 5-9.

One from located PD sources in transformer 1 is situated in the area of the tap changer. Characteristics belonged to the basic description of chosen signal, recorded in this area, are presented in Fig. 5. The signal is periodical, appears twice in the period of the supply voltage; it is located within very narrow phase ranges. Main frequency bands are: $110-130 \mathrm{kHz}$ and $140-150 \mathrm{kHz}$. In Fig. 6 there are presented basic characteristics within the band of 20 $50 \mathrm{kHz}$ for a such recorded signal. Main frequency band of this signal is $20-40 \mathrm{kHz}$, whereas remaining properties 


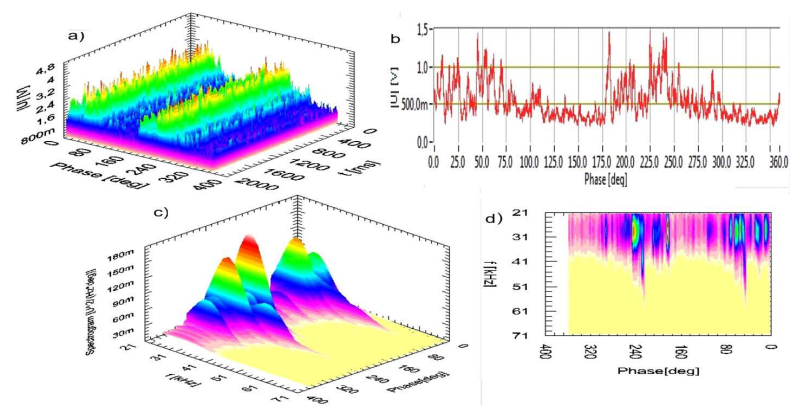

Fig. 3. Preliminary description of AE signal recorded at chosen measuring point of transformer 1 (PA032) with filtration within the band of 20-70 kHz: (a) phasetime characteristic, (b) averaging phase characteristic, (c) and (d) averaging short-time Fourier transform (STFT) spectrograms, $\mathrm{ADC}=-1.21$.

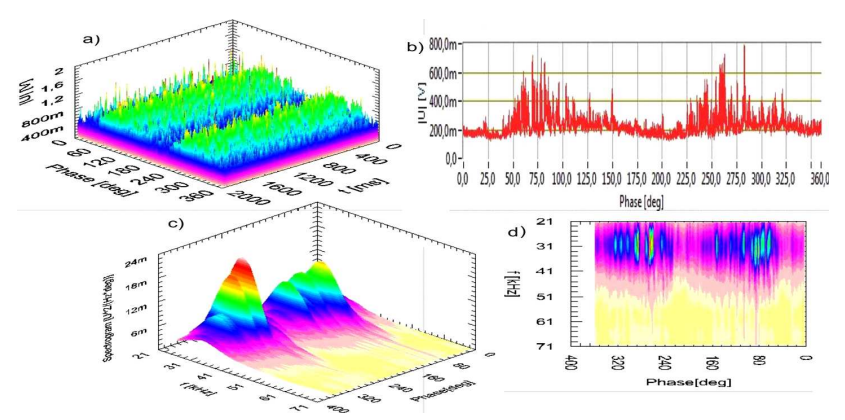

Fig. 4. Preliminary description of AE signal recorded at chosen measuring point of transformer 2 (J0102) with filtration within the band of $20-70 \mathrm{kHz}$ : (a) phase-time characteristic, (b) averaging phase characteristic, (c) and $(\mathrm{d})$ averaging STFT spectrograms, $\mathrm{ADC}=-0.65$.

are identical as ones of signal from Fig. 5. Effects connected with magnetic phenomena are insignificant in the area with the source and where an impulse generated by this source appears. Therefore characteristics within the band of $20-50 \mathrm{kHz}$ describe also only properties of signals coming form PD; it is visible that harmonic components of signal generated by PD appear within this band.

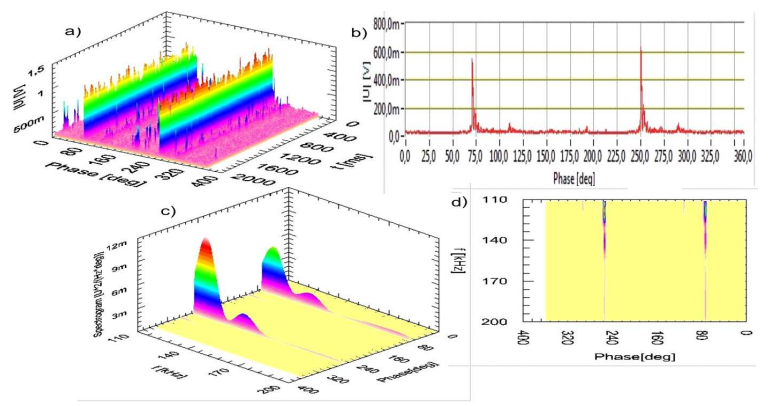

Fig. 5. Preliminary description of AE signal recorded at chosen measuring point of transformer 1 (PA072) with filtration within the band of $110-200 \mathrm{kHz}$ : (a) phase-time characteristic, (b) averaging phase characteristic, (c) and (d) averaging STFT spectrograms, $\mathrm{ADC}=-2.66$.

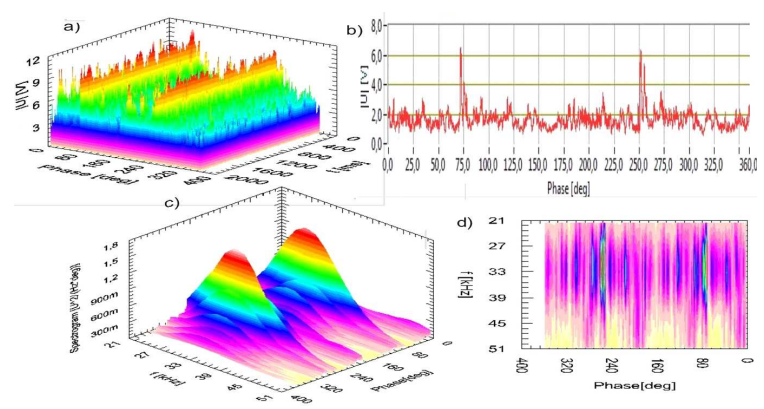

Fig. 6. Preliminary description of AE signal recorded at chosen measuring point of transformer 1 (PA072) with filtration within the band of $20-50 \mathrm{kHz}$ : (a) phasetime characteristic, (b) averaging phase characteristic, (c) and (d) averaging STFT spectrograms, ADC = -0.31 .

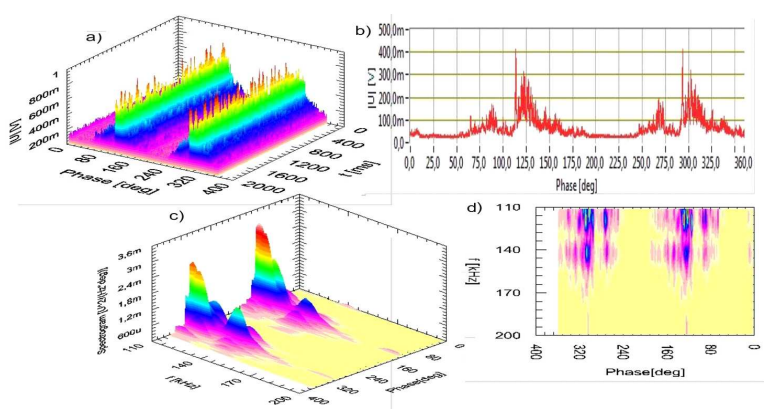

Fig. 7. Preliminary description of AE signal recorded at chosen measuring point of transformer 1 (PA0116) with filtration within the band of $110-200 \mathrm{kHz}$ : (a) phase-time characteristic, (b) averaging phase characteristic, (c) and (d) averaging STFT spectrograms, $\mathrm{ADC}=-4.72$.

Second PD source in transformer 1 appears from lower voltage side (horizontally $400-420 \mathrm{~cm}$, vertically 230 $250 \mathrm{~cm}$ ). Basic characteristics for signals coming from this source are presented in Fig. 7. Main frequency bands are identical as ones for a signal in Fig. 5, but location in the phase for these signals is other and ranges in which the signal is recorded are large.

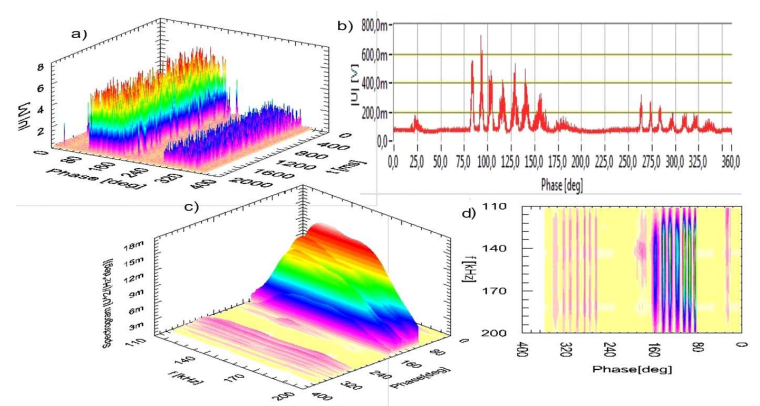

Fig. 8. Preliminary description of AE signal recorded at chosen measuring point of transformer 2 (J081) with filtration within the band of $110-200 \mathrm{kHz}$ : (a) phasetime characteristic, (b) averaging phase characteristic, (c) and (d) averaging STFT spectrograms, ADC = -0.34 . 


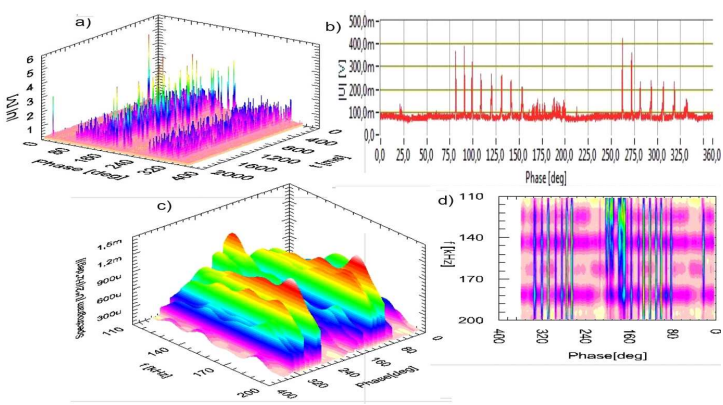

Fig. 9. Preliminary description of AE signal recorded at chosen measuring point of transformer 2 (J021) with filtration within the band of $110-200 \mathrm{kHz}$ : (a) phasetime characteristic, (b) averaging phase characteristic, (c) and (d) averaging STFT spectrograms, ADC = -0.60 .

Basic characteristics for two chosen PD signals within transformer 2 are presented in Figs. 8 and 9. The signal from Fig. 8 is generated by extensive PD source; it was recorded at measuring point in lower-voltage side. The signal from Fig. 9 was recorded at measuring point lying symmetrically in higher-voltage side. Averaging phase characteristics in Figs. 8b and 9b have similar structures of repeated peaks describing repeated $\mathrm{PD}$ which appear, go out and appear again (twice during one period of the supply voltage).

\section{Recapitulation}

In order to research AE signals coming from different acoustic sources in power oil transformers AE method has been applied.

Measuring band of $20-180 \mathrm{kHz}$ has been divided into the following bands: $20-70 \mathrm{kHz}, 60-120 \mathrm{kHz}, 110$ $180 \mathrm{kHz}$. Such a division enables us to distinguish signals coming from different acoustic sources:

- the band of $20-70 \mathrm{kHz}$ - PD sources and other acoustic phenomena,

- the band of $110-180 \mathrm{kHz}$ - PD sources.

Maps of descriptors in chosen frequency bands, worked up by kriging method, were used for location of different sources.

Investigation of two different transformers with identical construction but being in different state enables us to distinguish signals coming from partial discharges and being acoustic noise connected with inner acoustic interferences.

Detailed differentiation of signals coming from PD and other acoustic phenomena is effective by different properties with regard to time duration (phase band) and frequency characteristics.

Subsequent research in situ and model investigations leading to identification of different PD sources in power transformers are planned in the not distant future.

\section{Acknowledgments}

The researches were financed in the frame of the project N N505 466538 of Ministry of Science and Higher Education.

\section{References}

[1] F. Witos, Z. Gacek, A. Opilski, Arch. Acoust. 27, 65 (2002).

[2] F. Witos, Z. Gacek, P. Paduch, J. Phys. IV (France) 130, 179 (2006).

[3] F. Witos, Investigation of Partial Discharges by Means of Acoustic Emission Method and Electric Method, SUT, Gliwice 2008 (in Polish).

[4] F. Witos, Z. Gacek, Europ. Phys. J.-Spec. Top. 154, 239 (2008).

[5] F. Witos, Z. Gacek, Acta Phys. Pol. A 116, 422 (2009).

[6] F. Witos, A. Olszewska, Acta Phys. Pol. A 118, 1267 (2010).

[7] Z. Deheng, T. Kexiong, I. Xianche, in: II Conf. Properties and Application of Dielectric Mat., Ed. L. Zao, Vol. 2, Beijing 1988, p. 614.

[8] Y. Lu, X. Tan, X. Hu, IEE Proc. Sci. Meas. Technol. 147, 81 (2000).

[9] J. Deng, Opt. Laser Technol. 33, 305 (2001).

[10] T. Boczar, IEEE Trans. Diel. Electr. Insulat. 8, 598 (2001).

[11] M. MacAlpine, Z. Zhiquiang, M.S. Demokan, Electr. Power Syst. Res. 63, 27 (2002).

[12] T. Boczar, D. Zmarzly, IEEE Trans. Dielectr. Electr. Insulat. 11, 433 (2004).

[13] S.M. Markalous, IEEE Trans. Dielectr. Electr. Insulat. 15, 807 (2008).

[14] K. Gyung-Suk, I.-K. Kim, D.-W. Park, S.-Y. Choi, C.-Y. Park, Curr. Appl. Phys. 9, 296 (2009). 